\title{
Corynespora cassiicola causing leaf spots on Boehmeria nivea (rami) in Brazil
}

\author{
S. S. Salcedo ${ }^{1}$ - N. A. S. Ribeiro ${ }^{1}$ - A. L. Silva ${ }^{1}$ - A. A. Colman ${ }^{1}$ - R. W. Barreto ${ }^{1}$ \\ Received: 2 February 2019 / Accepted: 27 May 2019 /Published online: 11 June 2019 \\ (C) Australasian Plant Pathology Society Inc. 2019
}

\begin{abstract}
A new leaf spot disease caused by necrotrophic fungus Corynespora cassiicola is reported on Boehmeria nivea for the first time in Brazil. Identity was clarified based on morphological and molecular evidence and pathogenicity to ramie was demonstrated.
\end{abstract}

Keywords Ascomycota $\cdot$ Eye-spot $\cdot$ Dematiaceae $\cdot$ Fiber crop $\cdot$ Leaf disease $\cdot$ Necrotroph $\cdot$ Pathogenicity

Boehmeria nivea (Urticaceae) - common names ramie or China grass, rami in Brazil - is a perennial herb, native from tropical Asia, cultivated for fiber extraction for more than 7000 years, and also widely used as rabbit and goat fodder because of its high protein content (Simpson and ConnorOgorzaly 2001; Suryanah et al. 2017). It has always had great importance in China but its relevance is on the rise in countries such as Brazil, the Philippines, Japan, Taiwan, Indonesia, among others (Sen and Reddy 2011). Little is known about fungal diseases attacking ramie in Brazil. Only four fungi are listed in Farr and Rossman (2019) as occurring on ramie in Brazil. In Nov 2016, B. nivea adult individuals grown in a demonstration plot (Infectarium) in the campus of the Universidade Federal de Viçosa (state of Minas Gerais, Brazil) were observed bearing leaf spots of uncertain etiology. Either old or young leaves of adult and younger plants developed circular to irregular, 4-8 $\mathrm{mm}$ diameter necrotic dots. These turned dark brown, with concentric rings, reaching up to $20 \mathrm{~mm}$ diameter, with age (Figs. 1a-c). Samples were collected and taken to the laboratory for analysis. A representative sample of necrotic plants was dried in a plant press and deposited in the local herbarium at the Universidade Federal de Viçosa (Accession No VIC 44242).

A dematiaceus fungus was regularly found growing on the center of the ramie leaf spots. Homogeneous pure

S. S. Salcedo

taphrina10@gmail.com

1 Departamento de Fitopatologia, Universidade Federal de Viçosa, Viçosa, MG 36570-900, Brazil cultures were obtained by transferring single conidia from sporulating lesions onto potato dextrose-agar (PDA) plates with a sterile fine pointed needle. One representative isolate was deposited in the local culture collection of the Universidade Federal de Viçosa (Accession No COAD 1964).

Seven-day-old colonies grown at $25^{\circ} \mathrm{C}$ under a $12 \mathrm{~h}$ light regime in PDA were described following the terminology of Crous et al. (2009) and the color terminology of Rayner (1970). Fungal structures were scraped from lesions with a scalpel and slides were mounted in lactoglycerol and observed with a Olympus BX 51 light microscope fitted with differential interference contrast (DIC) illumination and connected to a Olympus Qcolor $3^{\mathrm{TM}}$ camera. The fungus had the following morphology: Conidiophores amphigenous, cylindrical, straight to slighlty curved, $132384 \times 35 \mu \mathrm{m}, 6-13$ septate, pale brown, smooth; conidia solitary or in short acropetal chains, cylindrical to subcylindrical, 92-154 × 3-6 $\mu \mathrm{m}, 10 \pm$ 20 pseudoseptate, pale brown, smooth (Fig. 1e and f). In culture: Fast-growing ( $7.8 \mathrm{~cm}$ diameter after 7 days in PDA), edges entire, umbonate, felty, pale vinaceous, bay reverse, not sporulating (Fig. 1d). Fungal morphology was equivalent to that of Corynespora cassiicola as described by Dixon et al. (2009) and Ellis and Holliday (1971).

To further confirm fungus identity, genomic DNA was extracted from 7- day old colonies using the Wizard Genomic DNA Purification Kit (Promega) by following the manufacturer's instructions. The ITS region of rRNA of the fungus was PCR amplified with ITS4 (TCC TCC GCT TAT TGA TAT GC) and ITS5 (GGA AGT AAA AGT CGT AAC AAG G) primers (White et al. 1990) and sequenced by Macrogen (Korea). The resulting sequence was deposited in GenBank (accession no. MH536840). This 
Fig. 1 Corynespora cassiicola on Boehmeria nivea. $\mathbf{a}, \mathbf{b}, \mathbf{c}$ Leaf spots showing concentric ring pattern. d Two week old colony on PDA plate. e Conidium. $\mathbf{f}$ Conidiophore showing enteroblastic percurrent proliferation. Bars $=20 \mu \mathrm{m}$
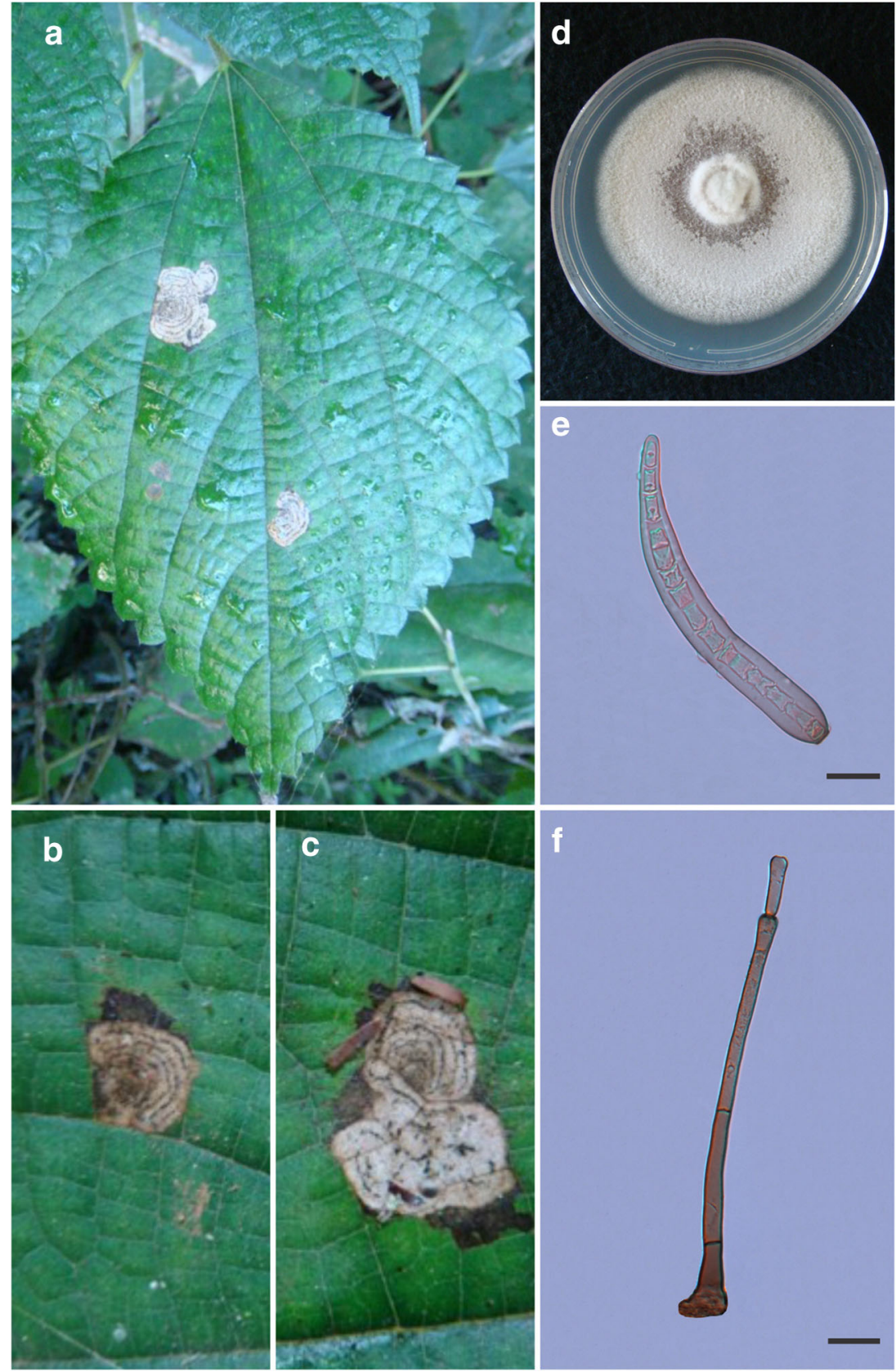

sequence had $99 \%$ identity with a previously deposited ITS sequence of $C$. cassiicola on Plectranthus barbatus (accession No. FJ852592) (Dixon et al. 2009).

A pathogenicity test was performed by inoculating five healthy six-month-old potted $B$. nivea with a $1 \times 10^{4}$ conidia/mL suspension of COAD 1964. Sporulating cultures were obtained on PDA after 10 days growth under black light $(40 \mathrm{~W})$ for $48-72 \mathrm{~h}$. Sporulating cultures were flooded with sterile water and scraped with a paintbrush to produce the conidial suspension, which was then adjusted to the correct concentration with the help of a haemocytometer. The conidial suspension was then sprayed on the abaxial surface of leaves until runoff. Plants were kept in a humid chamber, for 48 hs after inoculation, and then transferred to a greenhouse bench. Six days after inoculation leaf spot symptoms identical to those originally seen on the field appeared on inoculated plants, but not on controls - two healthy plants treated similarly but sprayed with distilled water. Corynespora cassiicola was isolated from necrotic tissues of inoculated plants and pure cultures of equivalent morphology to that COAD 1964 were obtained, and fungal structures mounted in lactoglycerol were observed confirming its identity as $C$. cassiicola, fulfilling Koch's postulates. 
Corynespora cassiicola is a common and cosmopolitan species, especially abundant in the tropics, with a wide range of host plants (Ellis and Holliday 1971; Dixon et al. 2009; Lopez et al. 2018). Farr and Rossman (2019) listed records of $C$. cassiicola in association with $B$. nivea from China and Malaysia but ramie is reported here for the first time as a host of $C$. cassiicola in Brazil.

\section{References}

Crous PW, Verkley JZ, Groenewald SRA (2009) CBS: fungal biodiversity. CBS-KNAW Fungal Biodiversity Centre, Utrecht

Dixon LJ, Schlub RL, Pernezny K, Datnoff LE (2009) Host specialization and phylogenetic diversity of Corynespora cassiicola. Phytopathology 99:1015-1027

Ellis MB, Holliday P (1971) Corynespora cassiicola. CMI Descr. pathog. Fungi Bact. 303:1-2
Farr DF, Rossman AY (2019) Fungal database, U. S. National Fungus Colletions, ARS, USDA. https://nt.ars-grin.gov/fungaldatabases/. Accessed 11 Jan 2019

Lopez D, Ribeiro S, Label P, Fumanal B, Venisse JS, Kohler A, de Oliveira RR, Labutti K, Lipzen A, Lail K, Bauer D, Ohm RA, Barry KW, Spatafora J, Grigoriev IV, Martin FM, Puiade- Renaud V (2018) Genome-wide analysis of Corynespora cassiicola leaf fall disease putative effectors. Front. Microbiol. 276:1-21

Rayner RW (1970) A mycological colour chart. Commonwealth mycological institute and British Mycological Society, Kew

Sen T, Reddy HNJ (2011) Various industrial applications of hemp, kinaf, flax and ramie natural fibres. Int J Innov Manage Technol 12:192-198

Simpson BB, Connor-Ogorzaly M (2001) Fibers, dyes, and tannins. In: Simpson BB, Connor-Ogorzaly M (eds) Economic botany: plants in our world. McGraw-Hill, New York, pp 355-377

Suryanah S, Rochana A, Susilawati I, Indiriani NP (2017) Ramie (Boehmeria nivea) plant nutrient quality as feed forage at various cutting ages. Anim. Prod. 19:111-117

White TJ, Bruns T, Lee S, Taylor J (1990) Amplification and direct sequencing of fungal ribosomal RNA genes for phylogenetics. In: Innis MA, Gelfand DH, Sninsky JJ (eds) PCR protocols: a guide to methods and amplifications. Academics Press, San Diego, pp 315-322 